\title{
Applications of Caristi's fixed point results
}

\author{
Abdul Latif*, Nawab Hussain and Marwan A Kutbi
}

* Correspondence: alatif@kau.edu. sa

Department of Mathematics, King Abdulaziz University, P.O. Box 80203, Jeddah 21589, Saudi Arabia

\begin{abstract}
In the setup of locally convex spaces, applying Caristi's results we prove some fixed point results for non-self multivalued maps and common fixed point theorems for Caristi type maps utilizing two different techniques. We apply our results to obtain some common fixed points for a Banach operator pair from the set of best approximations. Consequently, we either improve or extend a number of known results in the fixed point theory.

2000 Mathematics Subject Classification: 47H10; 54H25.
\end{abstract}

Keywords: fixed point, common fixed point; contraction map, Caristi's theorem, Banach operator pair, locally convex space

\section{Introduction}

One of the most useful generalizations of the Banach contraction principle in the setting of metric spaces is known as Caristi's fixed point theorem. In the past decades, Caristi's fixed point theorem has been generalized and extended in several directions (see, [1,2] and references therein). Applying this classical result, Massa [3], Yi and Zhao [4], Zhang [5], and Zhong et al. [6] and others proved fixed point theorems for non-self multivalued contraction maps in the setting of Banach spaces. There are spaces which are not normable (for example, see [7]). So there is natural and essential to study existence of fixed points in the setting of locally convex spaces. In fact, study of known fixed points results of Banach spaces to the case of locally convex spaces is neither trivial and nor easy. However, several interesting fixed point results for single valued and multivalued contraction and nonexpansive maps in the setting of locally convex spaces appeared in the literature, for example; see [8-15] and references there in.

In [16], Fang has introduced a notion of $F$-type topological spaces and generalized the Caristi's fixed point theorem to such topological spaces. Recently, Cammaroto et al. [17] observed that each Hausdorff locally convex topological vector space is an $F$ type topological space.

In [18], Chen and Li introduced the class of Banach operator pairs, as a new class of noncommuting maps and it has been further studied by Hussain [19,20], Hussain et al. [21], Khan and Akbar [22,23], and Pathak and Hussain [24].

In this article, applying Caristi's fixed point results and following the techniques in $[5,6]$, we prove some fixed point theorems for non-self multivalued contraction maps in the setup of locally convex spaces (see, Section 2). Consequently, Our results either improve or extend a number of known fixed point results including the corresponding results due to Massa [3], Yi and Zhao [4], Zhang [5], and Zhong et al. [6]. Section 3

(C) 2012 Latif et al.; licensee Springer. This is an open access article distributed under the terms of the Creative Commons Attribution License (http://creativecommons.org/licenses/by/2.0), which permits unrestricted use, distribution, and reproduction in any medium, provided the original work is properly cited. 
contains some general common fixed point theorems for Caristi type maps. Applying our theorems we derive some results on the existence of common fixed points for a Banach operator pair from the set of best approximations. Our results of Section 3 extend and unify the study of Al-Thagafi [25], Chen and Li [18], Hussain and Khan [11], Jungck and Sessa [26], Khan and Akbar [23], Pathak and Hussain [24] and many others.

\section{Fixed points for non-self multivalued maps}

In this section, $E$ denotes a complete Hausdorff locally convex topological vector space, $\mathcal{P}$ is the family of continuous seminorms generating the topology of $E$, and $K(E)$ is the family of nonempty compact subsets of $E$. For each $p \in \mathcal{P}$ and $A, B \in K(E)$, define

$$
D_{p}(A, B)=\max \left\{\sup _{a \in A} d_{p}(a, B), \sup _{b \in B} d_{p}(b, A)\right\}
$$

where $d_{p}(x, A)=\inf \{p(x-y): y \in A\}$ for any $x \in E$. It is known that $D_{p}$ is a metric on $K(E)$ even though $p$ is a seminorm, see [9,12]. Let $T: M \subset E \rightarrow K(E)$ be a multivalued map. We recall the following notions: (a) $T$ is called $\mathcal{P}$-contraction if for each $p \in \mathcal{P}$ there exists a constant $k_{p}, 0 \leq k_{p}<1$, such that $D_{p}(T(x), T(y)) \leq k_{p} p(x-y)$, for all $x, y \in M$. (b) A point $x \in M$ is called a fixed point of $T$ if and only if $x \in T(x)$. (c) We say $T$ satisfy the boundary condition $(\alpha)$ if for all $x \in M$ and all $y \in T(x),(x, y] \cap M \neq \emptyset$, where $(x, y]=\{(1-\lambda) x+\lambda y: 0<\lambda \leq 1\}$. (d) $T$ is called weakly inward if for each $x \in M, T(x) \subset \overline{I_{M}(x)}$, where $I_{M}(x)=\{z: z=x+\lambda(y-x), y \in M, \lambda \geq$ $1\}$ is known as the inward set.

Now, we state the Caristi's fixed point result in the setting of Hausdorff locally convex topological vector space, see $[17,16]$.

Theorem 2.1 Let $f: E \rightarrow E$ be any arbitrary map. Suppose there exists a lower semicontinuous function $\phi: E \rightarrow[0,+\infty)$ such that for each $x \in E$ and for each $p \in \mathcal{P}$,

$$
p(x-f(x)) \leq \varphi(x)-\varphi(f(x)) .
$$

Then $f$ has a fixed point.

Another generalization of the Caristi's fixed point result is the following which is a variant of Lemma 1.2 [6].

Theorem 2.2 Let $\phi: E \rightarrow[0,+\infty)$ be a bounded below lower semicontinuous function and $h:[0,+\infty) \rightarrow[0,+\infty)$ be a continuous nondecreasing function such that $\int_{0}^{+\infty} \frac{d r}{1+h(r)}=+\infty$. Let $f: E \rightarrow E$ be a map such that for any given $x_{0} \in E$ and for all $x \in$ E,

$$
\frac{p(x-f(x))}{1+h\left(p\left(x_{0}-x\right)\right)} \leq \varphi(x)-\varphi(f(x)) .
$$

Then $f$ has a fixed point.

Applying Theorem 2.1, first we prove the following fixed point result.

Theorem 2.3. Let $M$ be a nonempty closed subset of $E$ and $T: M \rightarrow K(E)$ be a $\mathcal{P}$-contraction map satisfying the boundary condition $(\alpha)$. Then $T$ has a fixed point.

Proof. Let $p \in \mathcal{P}$ be arbitrary and fixed. For each $x \in M$, choose $y \in T(x)$ such that $p$ $(x-y)=d_{p}(x, T(x))$. Set $z_{x p}$ as a farthest point from $x$ in $[x, y] \cap M$, that is; 


$$
p\left(x-z_{x p}\right)=\max \{p(x-w): w \in[x, y] \cap M\} .
$$

Then we have

$$
p(x-y)=p\left(x-z_{x p}\right)+p\left(z_{x p}-y\right) .
$$

Since $T$ is a compact valued $\mathcal{P}$-contraction map, then for $k_{p}, 0 \leq k_{p}<1$, we have

$$
\begin{aligned}
d_{p}\left(z_{x p}, T\left(z_{x p}\right)\right) & \leq p\left(z_{x p}-\gamma\right)+d_{p}\left(y, T\left(z_{x p}\right)\right) \\
& \leq p\left(z_{x p}-\gamma\right)+D_{p}\left(T(x), T\left(z_{x p}\right)\right) \\
& \leq p\left(z_{x p}-\gamma\right)+k_{p} p\left(x-z_{x p}\right) \\
& =p(x-y)-p\left(x-z_{x p}\right)+k_{p} p\left(x-z_{x p}\right) \\
& =d_{p}(x, T(x))-\left(1-k_{p}\right) p\left(x-z_{x p}\right) .
\end{aligned}
$$

Thus,

$$
p\left(x-z_{x p}\right) \leq\left(1-k_{p}\right)^{-1}\left\{d_{p}(x, T(x))-d_{p}\left(z_{x p}, T\left(z_{x p}\right)\right)\right\} .
$$

Define a self map $f$ on $M$ by $f_{p}(x)=z_{x p}, x \in M$, and define a nonnegative real valued function $\phi_{p}$ by $\phi_{p}(x)=\left(1-k_{p}\right)^{-1} d_{p}(x, T(x)), x \in M$. Then we have

$$
p\left(x-f_{p}(x)\right) \leq \varphi_{p}(x)-\varphi_{p}\left(f_{p}(x)\right) .
$$

Since $M$ is a closed subset of a complete space, so it is complete and hence by Theorem 2.1, $f$ has a fixed point $u \in M$. Note that $f_{p} u=u=z_{u p}$. Since, $z_{u p}$ is the farthest point from $u$ in $[u, y] \cap M$ and $u=z_{u p}$, so it follows that

$$
\left.d_{p}(u, T(u))\right)=p(u-\gamma)=0,
$$

and hence $u \in T(u)$.

Remark 2.4. Theorem 2.3 extends the fixed point result of Massa [[3], Theorem 2] to Hausdorff locally convex spaces.

Another application of Theorem 2.1, is the following fixed point result.

Theorem 2.5. Let $M$ be a closed subset of $E$ and $T: M \rightarrow K(E)$ be a $\mathcal{P}$-contraction map such that for all $x \in M$,

$$
\left\{z \in T(x): p(x-z)=d_{p}(x, T(x))\right\} \cap \overline{\mathrm{I}_{M}(x)} \neq \emptyset .
$$

Then $T$ has a fixed point.

Proof. Suppose that $T$ has no fixed point. Then, $d_{p}(x, T(x))>0$ for all $x \in M$. Choose $q \in(0,1)$ such that $k_{p}=k<\frac{1-q}{1+q}$, where $k \in(0,1)$. Since $T$ is a compact valued map, there exists $z \in T(x) \cap \overline{I_{M}(x)}$ such that

$$
d_{p}(x, T(x))=p(x-z)>0 .
$$

Then, there is some $t \in(0,1]$ such that

$$
t^{-1} d_{p}((1-t) x+t z, M)<q p(x-z) .
$$

Put $w=(1-t) x+t z$. Then there exists some $y \in M$, such that

$$
p(w-y)<q t p(x-z)=q p((1-t) x+t z-x)=q p(w-x) .
$$


Since

$$
p(y-x)-p(w-x) \leq p(w-y)<q p(w-x),
$$

it follows that

$$
p(y-x)<(1+q) p(w-x)
$$

and thus

$$
(a-1) p(w-x)<\frac{q-1}{q+1} p(x-y) .
$$

Note that

$$
p(w-x)+p(w-z)=p(x-z) .
$$

Since $T$ is a compact valued $\mathcal{P}$-contraction, we can choose $u \in T(x)$ and $v \in T(y)$ such that $p(w-u)=d_{p}(w, T(x))$ and

$$
p(u-v) \leq D_{p}(T(x), T(y)) \leq k p(x-y) .
$$

Now,

$$
\begin{aligned}
d_{p}(y, T(y)) & \leq p(y-v) \\
& \leq p(y-w)+p(w-u)+p(u-v) \\
& <q p(w-x)+d_{p}(w, T(x))+k p(x-y) \\
& <q p(w-x)+p(w-z)+k p(x-y) \\
& <q p(w-x)+p(x-z)-t p(x-z)+k p(x-y) \\
& <q p(w-x)+p(x-z)-p(w-x)+k p(x-\gamma) \\
& <(q-1) p(w-x)+p(x-z)+k p(x-\gamma) \\
& <\frac{q-1}{q+1} p(x-\gamma)+p(x-z)+k p(x-\gamma) \\
& <d_{p}(x, T(x))-\left(\frac{1-q}{1+q}-k\right) p(x-y)
\end{aligned}
$$

and thus we have

$$
d_{p}(y, T(y))<d_{p}(x, T(x))-c p(x-y)
$$

where $c=\frac{1-q}{1+q}-k$. Hence,

$$
p(x-y)<\frac{d_{p}(x, T(x))}{c}-\frac{d_{p}(y, T(y))}{c} .
$$

Define $f: M \rightarrow M$ by $f(x)=y$ and define the $\phi: M \rightarrow \mathbb{R}$ by $\varphi(x)=\frac{d_{p}(x, T(x))}{c}$. Clearly,

$$
p(x-f(x))<\varphi(x)-\varphi(f(x)) .
$$

By Theorem 2.1, $f$ has a fixed point $x_{0} \in M$. Thus, $f\left(x_{0}\right)=x_{0}$. On the other hand, we have

$$
0=p\left(x_{0}-f\left(x_{0}\right)\right)<\varphi\left(x_{0}\right)-\varphi\left(f\left(x_{0}\right)\right)=\varphi\left(x_{0}\right)-\varphi\left(x_{0}\right)=0,
$$


which is impossible. Hence, $T$ has a fixed point.

Corollary 2.6. Let $M$ be a closed subset of $E$ and let $T: M \rightarrow K(E)$ be a weakly inward $\mathcal{P}$-contraction map. Then $T$ has a fixed point.

Remark 2.7. (a) Theorem 2.5 extends the fixed point result of Zhang [[5], Theorem 3.3].

(b) It is worth to mention that in general, the contraction and weakly inward conditions of Theorem 2.5 can not be replaced with somewhat weaker conditions, namely, nonexpansive and $T(x) \cap \overline{I_{M}(x)} \neq \emptyset$, even in the setting of Banach spaces, see [5].

(c) Corollary 2.6 contains the fixed point result of Yi and Zhao [[4], Theorem 2.1].

Now, using Theorem 2.2 and a contractive condition basically due to [5], we prove the following fixed point result in the setting of Hausdorff locally convex topological vector spaces. Let $h:[0,+\infty) \rightarrow[0,+\infty)$ be a continuous nondecreasing function satisfying $\int_{0}^{+\infty} \frac{d r}{1+h(r)}=+\infty$.

Theorem 2.8. Let $M$ be a closed subset of $E$ and Let $T: M \rightarrow K(E)$ be a weakly inward map, $x_{0} \in M$, a given point and $\sigma \in(0,1]$ a constant. If for each $x, y \in M$,

$$
D_{p}(T(x), T(y)) \leq\left(1-\frac{\sigma}{1+h\left(p\left(x_{0}-x\right)\right)}\right) p(x-y) .
$$

Then $T$ has a fixed point.

Proof. Suppose that $T$ has no fixed point. Then, $d_{p}(x, T(x))>0$ for all $x \in M$. Choose $c, 0<c<\sigma$ and $q(x)=\frac{\sigma-c}{2\left(1+h\left(p\left(x_{0}-x\right)\right)\right)}$, then $0<q(x)<1$. Since $T$ is a compact valued map, there exists $z \in T(x)$ such that

$$
d_{p}(x, T(x))=p(x-z)>0 .
$$

Since $T$ is weakly inward, there exist $y \in M$ and $\lambda \geq 1$ such that $z=x+\lambda(y-x)$. Then,

$$
p(z-(x+\lambda(y-x)))<q(x) p(x-z) .
$$

Set $t=\frac{1}{\lambda}$ and $w=(1-t) x+t z$. Note that

$$
p(w-y)=\frac{1}{\lambda} p((\lambda-1) x+z-\lambda y)=t p(z-(x+\lambda(y-x))),
$$

thus we get

$$
\begin{array}{r}
p(w-y)<t q(x) p(x-z), \\
p(w-x)=t p(x-z), \text { and } p(w-z)=(1-t) p(x-z) .
\end{array}
$$

Also, we have

$$
p(x-y)<p(w-x)+t q(x) p(x-z)=(1+q(x)) p(w-x),
$$

and thus

$$
(q(x)-1) p(w-x)<\frac{q(x)-1}{q(x)+1} p(x-y),
$$


because $0<q(x)<1$. Since $T$ is a compact valued map, we can choose $u \in T(x)$ and $v$ $\in T(y)$ such that $p(w-u)=d_{p}(w, T(x))$ and

$$
p(u-v) \leq D_{p}(T(x), T(y)) .
$$

Now, using the above facts and the definition of $T$, we have

$$
\begin{aligned}
d_{p}(y, T(y)) & \leq p(y-v) \\
& \leq p(y-w)+p(w-u)+p(u-v) \\
& <q(x) p(w-x)+p(w-z)+D_{p}(T(x), T(y)) \\
& <\left(\frac{q(x)-1}{q(x)+1}+1\right) p(x-y)+p(x-z)-\frac{\sigma}{\left.1+h\left(p\left(x_{0}-x\right)\right) p\left(x_{0}-x\right)\right)} p(x-y) \\
& <2\left(\frac{\sigma-c}{2\left(1+h\left(p\left(x_{0}-x\right)\right)\right)}\right) p(x-y)+p(x-z)-\frac{\sigma}{1+h\left(p\left(x_{0}-x\right)\right)} p(x-y) \\
& <d_{p}(x, T(x))-\frac{c}{1+h\left(p\left(x_{0}-x\right)\right)} p(x-\gamma),
\end{aligned}
$$

and hence,

$$
\frac{p(x-y)}{1+h\left(p\left(x_{0}-x\right)\right)}<\frac{d_{p}(x, T(x))}{c}-\frac{d_{p}(y, T(y))}{c} .
$$

For any $x \in M$, define $f(x)=y$ and $\varphi(x)=\frac{d_{p}(x, T(x))}{c}$. Then, $f$ is a selfmap of $M$ and $\phi$ is a nonnegative real valued continuous function on $M$. Also, note that

$$
\frac{p(x-f(x))}{1+h\left(p\left(x_{0}-x\right)\right)} \leq \varphi(x)-\varphi(f(x)) .
$$

Applying Theorem 2.2, $f$ has a fixed point. But, due to the fact

$$
p(z-(x+\lambda(y-x)))<q(x) p(x-z)
$$

it follows that $f$ has no fixed point. This is a contradiction and hence $T$ has a fixed point.

Remark 2.9. a) Theorem 2.8 extends the fixed point result of [[6], Theorem 2.5] to the setting of Hausdorff locally convex spaces.

b) Theorem 2.8 is not true for multivalued nonexpansive maps, even in the setting of Banach spaces, see [6].

\section{Banach operator pair and Caristi type maps}

In this section, $(E, \tau)$ will be a Hausdorff locally convex topological vector space. A family $\left\{p_{\alpha}: \alpha \in I\right\}$ of seminorms defined on $E$ is said to be an associated family of seminorms for $\tau$ if the family $\{\gamma U: \gamma>0\}$, where $U=\bigcap_{i=1}^{n} U_{\alpha_{i}}$ and $U_{\alpha_{i}}=\left(x: p_{\alpha_{i}}(x)<1\right\}$, forms a base of neighborhoods of zero for $\tau$. A family $\left\{p_{\alpha}: \alpha \in\right.$ I\} of seminorms defined on $E$ is called an augmented associated family for $\tau$ if $\left\{p_{\alpha}: \alpha\right.$ $\in I\}$ is an associated family with property that the seminorm $\max \left\{p_{\alpha}, p_{\beta}\right\} \in\left\{p_{\alpha}: \alpha \in\right.$ I) for any $\alpha, \beta \in I$. The associated and augmented associated families of seminorms will be denoted by $A(\tau)$ and $A^{*}(\tau)$, respectively. It is well-known that given a locally convex space $(E, \tau)$, there always exists a family $\left\{p_{\alpha}: \alpha \in I\right\}$ of seminorms defined on $E$ such that $\left\{p_{\alpha}: \alpha \in I\right\}=A^{*}(\tau)$ (see $\left.[27,28]\right)$. 
The following construction will be crucial. Suppose that $M$ is a $\tau$-bounded subset of $E$. For this set $M$ we can select a number $\lambda_{\alpha}>0$ for each $\alpha \in I$ such that $M \subset \lambda_{\alpha} U_{\alpha}$ where $U_{\alpha}=\left\{x: p_{\alpha}(x) \leq 1\right\}$. Clearly $B=\cap_{\alpha} \lambda_{\alpha} \cap_{\alpha}$ is $\tau$-bounded, $\tau$-closed, absolutely convex and contains $M$. The linear span $E_{B}$ of $B$ in $E$ is $\bigcup_{n=1}^{\infty} n B$. The Minkowski functional of $B$ is a norm $\|\cdot\|_{B}$ on $E_{B}$. Thus $\left(E_{B},\|\cdot\|_{B}\right)$ is a normed space with $B$ as its closed unit ball and $\sup _{\alpha} p_{\alpha}\left(x / \lambda_{\alpha}\right)=\|\mathrm{x}\|_{B}$ for each $x \in E_{B}$ (for details see [27-30]).

In [31], Ciric introduced the following generalization of continuity for selfmaps.

Definition 3.1. A mapping $T$ of a topological space $X$ into itself is said to be orbitally continuous if $x_{0}, x \in X$ such that $\lim _{n \rightarrow \infty} T^{i_{n}}(x)=x_{0}$, then $\lim _{n \rightarrow \infty} T\left(T^{i_{n}}(x)\right)=T x_{0}$. (We shall say $T$ is orbitally continuous at $x_{0}$ (o.c.) if $x_{0}$ is such a point.)

Jungck [32] generalized the above definition as follows.

Definition 3.2. A mapping $T$ of a topological space $X$ into itself is said to be almost orbitally continuous (a.o.c.) at $x_{0} \in X$ if whenever $\lim _{n \rightarrow \infty} T^{i_{n}}(x)=x_{0}$ for some $x \in X$ and subsequence $\left\{T^{i_{n}}(x)\right\}$ of $\left\{T^{n}(x)\right\}$, there exists a subsequence $\left\{T^{j_{n}}(x)\right\}$ of $\left\{T^{n}(x)\right\}$ such that $\lim _{n \rightarrow \infty} T^{j_{n}}(x)=T x_{0}$. (If $T$ is a.o.c. at all $x \in M \subset X$, we say $T$ is (a.o.c.) on $M$; if $M=X, T$ is a.o.c.)

In [32], Jungck proved the following generalization of Caristi's Theorem (see, Theorem 1.2 [33]) which will be needed in the sequel.

Theorem 3.3. Let $(X, d)$ be a complete metric space and $S, T$ be two a.o.c. mappings of $X$ into itself. Suppose that there are a finite number of functions $\left\{\varphi_{i}: 1 \leq i \leq n_{0}\right\}$ of $X$ into $[0, \infty)$ such that

$$
d(S x, T y) \leq k d(x, y)+\sum_{i=1}^{n_{0}}\left[\phi_{i}(x)-\phi_{i}(S x)+\phi_{i}(y)-\phi_{i}(T y)\right]
$$

for all $x, y \in X$ and some $k \in[0,1)$. Then $S$ and $T$ have a common fixed point $x_{0} \in$ $X$. Further, if $x \in X$, then $S^{n} x \rightarrow x_{0}$ and $T^{n} x \rightarrow x_{0}$ as $n \rightarrow \infty$.

The pair $(T, f)$ of selfmaps of $M$ is called a Banach operator pair, if the set $F(f)$ is $T$ invariant, namely $T(F(f)) \subseteq F(f)$. Obviously, commuting pair $(T, f)$ is a Banach operator pair but converse is not true, in general; see [18,24]. A mapping $T: M \rightarrow E$ is called demiclosed at 0 if $\left\{x_{\alpha}\right\}$ converges weakly to $x$ and $\left\{T x_{\alpha}\right\}$ converges to 0 , then we have $T x=0$.

The aim of this section is to extend the above mentioned result of Jungck to locally convex spaces and establish general common fixed point theorems for Caristi type maps in the setting of a locally convex space. We apply our theorems to derive some results on the existence of common fixed points for a Banach operator pair from the set of best approximations. Our results extend and unify the study of Al-Thagafi [25], Chen and Li [18], Hussain and Khan [11], Jungck and Sessa [26], Khan and Akbar [23], and Pathak and Hussain [24] and many others.

We observe in the following example that the almost orbital continuity of a selfmap $T$ on a metric space depends on the choice of the metric. Here, $\omega=\mathbf{N} \cup\{0\}$ and $\mathbf{N}$ is the set of positive integers.

$$
\text { Example 3.4. Let } X=\{0\} \bigcup\left\{\frac{1}{2^{n}}: n \in \omega\right\} \text { and } T(0)=1, T\left(\frac{1}{2^{n}}\right)=\frac{1}{2^{n+1}} \text { for } n \in \omega \text {. }
$$

Then $T$ is not (a.o.c.) under usual metric on $X([32]$, Example 4.2) but $T$ is continuous, and therefore (a.o.c.) under the discrete metric on $X$. 
Next, we establish a positive result in this direction in the context of linear topologies utilizing Minkowski functional.

Lemma 3.5. Let $T$ be (a.o.c.) selfmap of a $\tau$-bounded subset $M$ of a Hausdorff locally convex space $(E, \tau)$. Then $T$ is (a.o.c.) on $M$ with respect to $\|\cdot\|_{B}$.

Proof. By hypothesis, there exists a subsequence $\left\{T^{j_{n}}(x)\right\}$ of $\left\{T^{n}(x)\right\}$ such that $\lim _{n \rightarrow \infty} p_{\alpha}\left(T^{j_{n}}(x)-T x_{0}\right)=0$ for each $p_{\alpha} \in A^{*}(\tau)$ for some $x \in X$, whenever $\lim _{n \rightarrow \infty} p_{\alpha}\left(T^{i_{n}}(x)-x_{0}\right)=0$ for each $p_{\alpha} \in A^{*}(\tau)$ for some $x \in X$ and subsequence $\left\{T^{i_{n}}(x)\right\}$ of $\left\{T^{n}(x)\right\}$. Taking supremum on both sides, we get

$$
\sup _{\alpha} \lim _{n \rightarrow \infty} p_{\alpha}\left(\frac{T^{j_{n}}(x)-T x_{0}}{\lambda_{\alpha}}\right)=\sup _{\alpha}\left(\frac{0}{\lambda_{\alpha}}\right)
$$

whenever

$$
\sup _{\alpha} \lim _{n \rightarrow \infty} p_{\alpha}\left(\frac{T^{i_{n}}(x)-x_{0}}{\lambda_{\alpha}}\right)=\sup _{\alpha}\left(\frac{0}{\lambda_{\alpha}}\right) .
$$

This implies that

$$
\lim _{n \rightarrow \infty} \sup _{\alpha} p_{\alpha}\left(\frac{T^{j_{n}}(x)-T x_{0}}{\lambda_{\alpha}}\right)=0
$$

whenever

$$
\lim _{n \rightarrow \infty} \sup _{\alpha} p_{\alpha}\left(\frac{T^{i_{n}}(x)-x_{0}}{\lambda_{\alpha}}\right)=0 .
$$

Hence there exists a subsequence $\left\{T^{j_{n}}(x)\right\}$ of $\left\{T^{n}(x)\right\}$ such that $\lim _{n \rightarrow \infty}\left\|T^{j_{n}}(x)-T x_{0}\right\|_{B}=0$, whenever $\lim _{n \rightarrow \infty}\left\|T^{i_{n}}(x)-x_{0}\right\|_{B}=0$ for some $x \in X$ and subsequence $\left\{T^{i_{n}}(x)\right\}$ of $\left\{T^{n}(x)\right\}$ as desired.

An application of Lemma 3.5 provides the following general Caristi's Theorem in the setting of locally convex space.

Theorem 3.6. Let $M$ be a nonempty $\tau$-bounded, $\tau$-sequentially complete subset of a Hausdorff locally convex space $(E, \tau)$ and $S, T$ be two almost orbitally continuous mappings of $M$ into itself. Suppose that there are a finite number of functions $\left\{\varphi_{i}: 1 \leq i \leq\right.$ $\left.n_{0}\right\}$ of $M$ into $[0, \infty)$ such that

$$
p_{\alpha}(S x-T y) \leq k p_{\alpha}(x-y)+\sum_{i=1}^{n_{0}}\left[\phi_{i}(x)-\phi_{i}(S x)+\phi_{i}(T y)\right]
$$

for all $x, y \in X, p_{\alpha} \in A^{*}(\tau)$ and some $k \in[0,1)$. Then $S$ and $T$ have a common fixed point $x_{0} \in X$. Further, if $x \in X$, then $S^{n} x \rightarrow x_{0}$ and $T^{n} x \rightarrow x_{0}$ as $n \rightarrow \infty$.

Proof. Since the norm topology on $E_{B}$ has a base of neighborhoods of 0 consisting of $\tau$-closed sets and $\mathrm{M}$ is $\tau$-sequentially complete, therefore $\mathrm{M}$ is $\|\cdot\|_{B^{-}}$sequentially complete in $\left(E_{B},\|\cdot\|_{B}\right)$ (see, $\left.[11,29,30]\right)$. By Lemma $3.5, S, T$ are $\|\cdot\|_{B^{-}}$almost orbitally continuous mappings of $M$. From (4.1) we obtain for any $x, y \in M$,

$$
\sup _{\alpha} p_{\alpha}\left(\frac{S x-T y}{\lambda_{\alpha}}\right) \leq k \sup _{\alpha} p_{\alpha}\left(\frac{x-y}{\lambda_{\alpha}}\right)+\sum_{i=1}^{n_{0}}\left[\phi_{i}(x)-\phi_{i}(S x)+\phi_{i}(y)-\phi_{i}(T y)\right] .
$$


Thus,

$$
\|S x-T y\|_{B} \leq k\|x-y\|_{B}+\sum_{i=1}^{n_{0}}\left[\phi_{i}(x)-\phi_{i}(S x)+\phi_{i}(y)-\phi_{i}(T y)\right] .
$$

A comparison of our hypothesis with that of Theorem 3.3 tells that we can apply it to $M$ as a subset of $\left(E_{B},\|\cdot\|_{B}\right)$ to conclude that there exists a point $z$ in $M$ such that $T z$ $=S z=z$ and if $x \in M$, then $S^{n} x \rightarrow z$ and $T^{n} x \rightarrow z$ as $n \rightarrow \infty$.

Lemma 3.7. Let $M$ be a nonempty $\tau$-bounded subset of Hausdorff locally convex space (E, $\tau), S, T$ and $f$ be self-maps of $M$ and $S, T$ be (a.o.c). Suppose that there are a finite number of functions $\left\{\varphi_{i}: 1 \leq i \leq n_{0}\right\}$ of $M$ into $[0, \infty)$ such that

$$
p_{\alpha}(S x-T y) \leq k p_{\alpha}(f x-f y)+\sum_{i=1}^{n_{0}}\left[\phi_{i}(x)-\phi_{i}(S x)+\phi_{i}(f y)-\phi_{i}(T y)\right]
$$

for all $x, y \in X, p_{\alpha} \in A^{*}(\tau)$ and some $k \in[0,1)$. If $F(f)$ is nonempty and $\tau$-sequentially complete and $\tau-c l(T(F(f))) \subseteq F(f)$ and $\tau-c l(S(F(f))) \subseteq F(f)$. Then, $M \cap F(S) \cap F(T) \cap F(f) \neq \varnothing$.

Proof. Note that for all $x, y \in F(f)$, we have,

$$
\begin{aligned}
p_{\alpha}(S x-T y) & \leq k p_{\alpha}(f x-f y)+\sum_{i=1}^{n_{0}}\left[\phi_{i}(f x)-\phi_{i}(S x)+\phi_{i}(f y)-\phi_{i}(T y)\right] \\
& =k p_{\alpha}(x-y)+\sum_{i=1}^{n_{0}}\left[\phi_{i}(x)-\phi_{i}(S x)+\phi_{i}(y)-\phi_{i}(T y)\right]
\end{aligned}
$$

Hence $S$, $T$ satisfy (3.1) on $F(f)$ and $\tau-c l(T(F(f))) \subseteq F(f)$, and $\tau-c l(S(F(f)) \subseteq F(f)$. By Theorem 3.6, $S, T$ have a fixed point $z$ in $F(f)$ and consequently $F(S) \cap F(T) \cap F(f) \neq 0$.

Corollary 3.8. Let $M$ be a nonempty $\tau$-bounded subset of Hausdorff locally convex space $(E, \tau), S, T$, and $f$ be self-maps of $M$ and $S, T$ be (a.o.c). Suppose that there are a finite number of functions $\left\{\varphi_{i}: 1 \leq i \leq n_{0}\right\}$ of $M$ into $[0, \infty)$ such that

$$
p_{\alpha}(T x-T y) \leq k p_{\alpha}(f x-f y)+\sum_{i=1}^{n_{0}}\left[\phi_{i}(f x)-\phi_{i}(T x)+\phi_{i}(f y)-\phi_{i}(T y)\right]
$$

for all $x, y \in X, p_{\alpha} \in A^{*}(\tau)$ and some $k \in[0,1)$. If $F(f)$ is nonempty $\tau$-sequentially complete and $(T, f)$ is a Banach operator pair. Then, $M \cap F(S) \cap F(T) \cap F(f) \neq \varnothing$.

The following result generalizes [[18], Theorems 3.2, 3.3] and improves [[25], Theorem 2.2], and [[26], Theorem 6]. Notice that $[q, T x]=\{(1-k) q+k T x: k \in[0,1]\}$.

Theorem 3.9. Let $M$ be a nonempty $\tau$-bounded subset of Hausdorff locally convex [resp., complete] space $(E, \tau)$ and $T, f$ be self-maps of $M$. Suppose that $T$ is continuous, $F(f)$ is $q$-starshaped, $\tau$-closed [resp., $\tau$-weakly closed], $\tau-c l(T(F(f))) \subseteq F(f)$ [resp., $\tau$ - wcl $(T(F(f)) \subseteq F(f)], M$ is $\tau$-compact [resp., $M$ is weakly $\tau$-compact, $I$ - $T$ is demiclosed at 0 , where $I$ stands for identity map]. Assume that there are a finite number of functions $\left\{\varphi_{i}: 1 \leq i \leq n_{0}\right\}$ of $M$ into $[0, \infty)$ such that

$$
p_{\alpha}(T x-T y) \leq p_{\alpha}(f x-f y)+\frac{1}{k} \sum_{i=1}^{n_{0}}\left[\phi_{i}(f x)-\phi_{i}\left(q_{x}\right)+\phi_{i}(f y)-\phi_{i}\left(q_{y}\right)\right],
$$

for all $x, y \in M, q_{x} \in[q, T x], q_{y} \in[q, T y]$ and $k \in(0,1)$. Then $M \cap F(T) \cap F(f) \neq \emptyset$.

Proof. Define $T_{n}: F(f) \rightarrow F(f)$ by $T_{n} x=\left(1-k_{n}\right) q+k_{n} T x$ for all $x \in F(f)$ and a fixed sequence of real numbers $k_{n}\left(0<k_{n}<1\right)$ converging to 1 . Since $F(f)$ is $q$-starshaped and $\tau-c l(T(F(\mathrm{f}))) \subseteq F(f)[$ resp., $\tau-w c l(T(F(f))) \subseteq F(f)]$, so $\left.\tau-c l\left(T_{n}(F(f))\right) \subseteq F(f)\right]$ [resp., $\tau$ $\left.w c l\left(T_{n}(F(f))\right) \subseteq F(f)\right]$ for each $n \geq 1$. 
Also by (3.3),

$$
\begin{aligned}
p_{\alpha}\left(T_{n} x-T_{n} y\right) & =k_{n} p_{\alpha}(T x-T y) \\
& \leq k_{n}\left(p_{\alpha}(f x-f y)+\frac{1}{k_{n}} \sum_{i=1}^{n_{0}}\left[\phi_{i}(f x)-\phi_{i}\left(T_{n} x\right)+\phi_{i}(f y)-\phi_{i}\left(T_{n} y\right)\right]\right), \\
& \leq k_{n} p_{\alpha}(f x-f y)+\sum_{i=1}^{n_{0}}\left[\phi_{i}(f x)-\phi_{i}\left(T_{n} x\right)+\phi_{i}(f y)-\phi_{i}\left(T_{n} y\right)\right],
\end{aligned}
$$

for each $x, y \in F(f)$ and some $0<k_{n}<1$.

If $M$ is $\tau$-compact so is $\tau$-sequentially complete. By Lemma 3.7, for each $n \geq 1$, there exists $x_{n} \in F(f)$ such that $x_{n}=f x_{n}=T_{n} x_{n}$. The compactness of $\tau-\operatorname{cl}(M)$ implies that there exists a subsequence $\left\{T x_{m}\right\}$ of $\left\{T x_{n}\right\}$ such that $T x_{m} \rightarrow z \in \operatorname{cl}(M)$ as $m \rightarrow \infty$. Since $\left\{T x_{m}\right\}$ is a sequence in $T(F(f))$ and $\tau-c l(T(F(f))) \subseteq F(f)$, therefore $z \in F(f)$. Further, $x_{m}=T_{m} x_{m}=\left(1-k_{m}\right) q+k_{m} T x_{m} \rightarrow z$. By the continuity of $T$, we obtain $T z=$ $z$. Thus, $M \cap F(T) \cap F(f) \neq \varnothing$ proves the first case.

By Lemma 3.7, for each $n \geq 1$, there exists $x_{n} \in F(f)$ such that $x_{n}=f x_{n}=T_{n} x_{n}$. Moreover, we have $p_{\alpha}\left(x_{n}-T x_{n}\right) \rightarrow 0$ as $n \rightarrow \infty$. The weak $\tau$-compactness of $M$ implies that there is a subsequence $\left\{T x_{m}\right\}$ of $\left\{T x_{n}\right\}$ converging weakly to $y \in M$ as $m \rightarrow \infty$. Since $\left\{T x_{m}\right\}$ is a sequence in $T(F(f))$, therefore $y \in \tau-w c l(T(F(f))) \subseteq F(f)$. Also we have, $x_{m}$ $T x_{m} \rightarrow 0$ as $m \rightarrow \infty$. If $I-T$ is demiclosed at 0 , then $y=T y$. Thus, $M \cap F(T) \cap F(f) \neq \varnothing$.

If $\sum_{i=1}^{n_{0}}\left[\phi_{i}(f x)-\phi_{i}\left(q_{x}\right)+\phi_{i}(f y)-\phi_{i}\left(q_{y}\right)\right] \geq 0$ in (3.4), then every $f$-nonexpansive map $T$ satisfies (3.4). Thus we obtain the following result.

Corollary 3.10. Let $M$ be a nonempty $\tau$-bounded subset of Hausdorff locally convex [resp., complete] space $(E, \tau)$ and $T, f$ be self-maps of $M$. Suppose that $T$ is continuous, $F(f)$ is $q$-starshaped, $\tau$-closed [resp, $\tau$-weakly closed], $\tau-c l(T(F(f))) \subseteq F(f)$ [resp, $\tau-$ wcl $(T(F(f))) \subseteq F(f)$ ], $M$ is $\tau$-compact [resp, $M$ is weakly $\tau$-compact, $I-T$ is demiclosed at $0]$. Assume that there are a finite number of functions $\left\{\varphi_{i}: 1 \leq i \leq n_{0}\right\}$ of $M$ into [0, $\infty)$ such that $\sum_{i=1}^{n_{0}}\left[\phi_{i}(f x)-\phi_{i}\left(q_{x}\right)+\phi_{i}(f y)-\phi_{i}\left(q_{y}\right)\right] \geq 0$ for all $x, y \in M, q_{x} \in[q, T x]$, $q_{y} \in[q, T y]$. If $T$ is $f$-nonexpansive, then $M \cap F(T) \cap F(f) \neq \varnothing$.

Corollary 3.11. Let $M$ be a nonempty $\tau$-bounded subset of Hausdorff locally convex [resp., complete] space $(E, \tau)$ and $T, f$ be self-maps of $M$. Suppose that $T$ is continuous, $F(f)$ is $q$-starshaped, and $\tau$-closed [resp., $\tau$-weakly closed], $M$ is $\tau$-compact [resp., $M$ is weakly $\tau$-compact, $I-T$ is demiclosed at 0$],(T, f)$ is a Banach operator pair and satisfy (3.4) for all $x, y \in M$. Then $M \cap F(T) \cap F(f) \neq 0$.

We define $P_{M}(u)=\left\{y \in M: p_{\alpha}(y-u)=d_{p_{\alpha}}(u, M)\right.$, for all $\left.p_{\alpha} \in A^{*}(\tau)\right\}$ and denote by $\mathfrak{I}_{0}$ the class of closed convex subsets of $E$ containing 0 . For $M \in \mathfrak{I}_{0}$, we define $M_{u}=\{x$ $\in M: p_{\alpha}(x) \leq 2 p_{\alpha}(u)$ for each $\left.p_{\alpha} \in A^{*}(\tau)\right\}$. It is clear that $P_{M}(u) \subset M_{u} \in \Im_{0}$.

The following result extends [[25], Theorem 4.1] and [[34], Theorem 2.14] and corresponding results in [24].

Theorem 3.12. Let $f, T$ be self-maps of a a Hausdorff locally convex space $E$. If $u \in$ $E$ and $M \in \Im_{0}$ such that $T\left(M_{u}\right) \subseteq M, \tau-\operatorname{cl}\left(T\left(M_{u}\right)\right)$ is compact and $\|T x-u\| \leq\|x-u\|$ for all $x \in M_{u}$, then $P_{M}(u)$ is nonempty, closed and convex with $T\left(P_{M}(u)\right) \subseteq P_{M}(u)$. If, in addition, $D \subseteq P_{M}(u), D_{0}:=D \cap F(f)$ is $q$-starshaped, $\tau$-closed, $\tau-\operatorname{cl}\left(T\left(D_{0}\right)\right) \subseteq D_{0}, T$ is continuous on $D$ and (4.3) holds for all $x, y \in D$, then $P_{M}(u) \cap F(T) \cap F(f) \neq \emptyset$.

Proof. Follows the lines of proof of Theorem 3.9 [8], so is omitted. 
Remark 3.13. It is worth to mention that our results are nontrivial generalizations of the corresponding known fixed point results in the setting of Banach spaces because there are plenty of spaces which are not normable (see, [[7], p. 113]). So it is natural to consider fixed point and approximation results in the context of locally convex spaces.

\author{
Acknowledgements \\ The authors gratefully acknowledge the financial support from the Deanship of Scientific Research (DSR) at King \\ Abdulaziz University (KAU) represented by the Unit of Research Groups through the grant number $(11 / 31 / \mathrm{Gr})$ for the \\ group entitled "Nonlinear Analysis and Applied Mathematics".
}

Authors' contributions

All the authors contributed equally. All authors read and approved the final manuscript.

\title{
Competing interests
}

The authors declare that they have no competing interests.

Received: 23 October 2011 Accepted: 21 February 2012 Published: 21 February 2012

\section{References}

1. Agarwal, RP, Khamsi, MA: Extension of Caristi's fixed point theorem to vector valued metric space. Nonlinear Anal. 74, 141-145 (2011). doi:10.1016/j.na.2010.08.025

2. Khamsi, MA: Remarks on Caristi's fixed point theorem. Nonlinear Anal. 71, 227-231 (2009). doi:10.1016/j.na.2008.10.042

3. Massa, S: Some remarks on Opial spaces. Boll Un Mat Ital. 6, 65-70 (1983)

4. Yi, HW, Zhao, YC: Fixed point theorems for weakly inward multivalued mappings and their randomizations. J Math Anal Appl. 183, 613-619 (1994). doi:10.1006/jmaa.1994.1167

5. Zhang, S: Star-shaped sets and fixed points of multivalued mappings. Math Japon. 36(2):327-334 (1991)

6. Zhong, CK, Zhu, J, Zhao, PH: An extension of multi-valued contraction mappings and fixed points. Proc Am Math Soc. 128(8):2439-2444 (1999)

7. Fabian, M, Habala, P, Hajek, P, Montesinos Santalucia, V, Pelant, J, Zizler, V: Functional Analysis and Infinite Dimentional Geometry, CMS Books in Mathematics. Springer, New York (2001)

8. Akbar, F, Khan, AR: Common fixed point and approximation results for noncommuting maps on locally convex spaces. Fixed Point Theory and Applications 2009(2009):14 (2009). Article ID 207503

9. Anderson, DE, Nelson, JL, Singh, KL: Fixed points for single and multivalued mappings in locally convex spaces. Math Japon. 31(5):665-672 (1986)

10. Caristi, J: Fixed point theorems for mappings satisfying inwardness conditions. Trans Am Math Soc. 215, $241-251$ (1976)

11. Hussain, N, Khan, AR: Common fixed point results in best approximation theory. Appl Math Lett. 16, 575-580 (2003) doi:10.1016/S0893-9659(03)00039-9

12. Ko, HM, Tsai, YH: Fixed point theorems for point to set mappings in locally convex spaces and acharacterization of complete metric spaces. Bull Acad Sinica. 7, 461-470 (1979)

13. O'Regan, D: Fixed point for set-valued mappings in locally convex linear topological spaces. Math Comput Model. 28, 45-55 (1998)

14. Singh, KL, Chen, Y: Fixed points for nonexpansive multivalued mapping in locally convex spaces. Math Japon. 36 423-425 (1991)

15. Su, CH, Sehgal, VM: Some fixed point theorems for nonexpansive multivalued functions in locally convex spaces. Acad Sinica. 4, 49-52 (1976)

16. Fang, J: The variational principle and fixed point theorems in certain topological spaces. J Math Anal Appl. 202, 398-412 (1996). doi:10.1006/jmaa.1996.0323

17. Cammaroto, F, Chinni, A, Sturiale, G: A remark on Ekeland's principle in locally convex topological vector spaces. Math Comput Model. 30, 75-79 (1999)

18. Chen, J, Li, Z: Common fixed points for Banach operator pairs in best approximation. J Math Anal Appl. 336, 1466-1475 (2007). doi:10.1016/j.jmaa.2007.01.064

19. Hussain, N: Asymptotically pseudo-contractions, Banach operator pairs and best simultaneous approximations. Fixed Point Theory and Applications 2011, 11 (2011). Article ID 812813. doi:10.1186/1687-1812-2011-11

20. Hussain, N: Common fixed points in best approximation for Banach operator pairs with Ćirić type I-contractions. J Math Anal Appl. 338, 1351-1362 (2008). doi:10.1016/j.jmaa.2007.06.008

21. Hussain, N, Khamsi, MA, Latif, A: Banach operator pairs and common fixed points in hyperconvex metric spaces. Nonlinear Anal. 743, 5956-5961 (2011)

22. Khan, AR, Akbar, F: Best simultaneous approximations, asymptotically nonexpansive mappings and variational inequalities in Banach spaces. J Math Anal Appl. 354, 469-477 (2009). doi:10.1016/j.jmaa.2009.01.007

23. Khan, AR, Akbar, F: Common fixed points from best simultaneous approximations. Tiawanese J Math. 13, 1379-1386 (2009)

24. Pathak, HK, Hussain, N: Common fixed points for Banach operator pairs with applications. Nonlinear Anal. 69, 2788-2802 (2008). doi:10.1016/j.na.2007.08.051

25. Al-Thagafi, MA: Common fixed points and best approximation. J Approx Theory. 85, 318-323 (1996). doi:10.1006/ jath.1996.0045

26. Jungck, G, Sessa, S: Fixed point theorems in best approximation theory. Math Japon. 42, 249-252 (1995) 
27. Hamel, AA: Phelp's lemma, Dane's drop theorem and Ekeland's principle in locally convex spaces. Proc Am Math Soc. 131, 3025-3038 (2003). doi:10.1090/S0002-9939-03-07066-7

28. Kothe, G: Topological Vector Spaces. I. Springer-Verlag, New York159 (1969)

29. Lixin, C, Yunchi, Z, Fong, Z: Dane's drop theorem in locally convex spaces. Proc Am Math Soc. 124, 3699-3702 (1996). doi:10.1090/50002-9939-96-03404-1

30. Tarafdar, E: Some fixed-point theorems on locally convex linear topological spaces. Bull Austral Math Soc. 13, 241-254 (1975). doi:10.1017/S0004972700024436

31. Cirić, LB: A generalized contractions and fixed point theorems. Publ Inst Math (Beograd). 12, 19-26 (1971)

32. Jungck, GF: Generalizations of continuity in the context of proper orbits and fixed pont theory. Topol Proc. 37, 1-15 (2011)

33. Dien, NH: Some remarks on common fixed point theorems. J Math Anal Appl. 187, 76-90 (1994). doi:10.1006/ jmaa.1994.1345

34. Hussain, N, Jungck, GF: Common fixed point and invariant approximation results for noncommuting generalized (f,g)nonexpansive maps. J Math Anal Appl. 321, 851-861 (2006). doi:10.1016/j.jmaa.2005.08.045

doi:10.1186/1029-242X-2012-40

Cite this article as: Latif et al: Applications of Caristi's fixed point results. Journal of Inequalities and Applications 2012 2012:40.

\section{Submit your manuscript to a SpringerOpen ${ }^{\circ}$} journal and benefit from:

- Convenient online submission

- Rigorous peer review

- Immediate publication on acceptance

- Open access: articles freely available online

- High visibility within the field

- Retaining the copyright to your article

Submit your next manuscript at $\boldsymbol{s p r i n g e r o p e n . c o m ~}$ 